Original Research Paper

\title{
Classifying and Predicting Students' Performance using Improved Decision Tree C4.5 in Higher Education Institutes
}

\author{
${ }^{1}$ Mohammed Hikmat Sadiq and ${ }^{2}$ Nawzat Sadiq Ahmed \\ ${ }^{1}$ Department of Computer Science, University of Duhok, Duhok, Kurdistan Region, \\ Iraq and University of Zakho, Duhok, Kurdistan Region, Iraq \\ ${ }^{2}$ Directorate of General Registration, Duhok Polytechnic University, Kurdistan Region, Duhok, Iraq
}

\author{
Article history \\ Received: 16-05-2019 \\ Revised: 05-08-2019 \\ Accepted: 11-09-2019 \\ Corresponding Author: \\ Mohammed Hikmat Sadiq \\ Department of Computer \\ Science, University of Duhok, \\ Duhok, Kurdistan Region, Iraq \\ and University of Zakho, \\ Duhok, Kurdistan Region, Iraq \\ Email: hamamezory@gmail.com
}

\section{Introduction}

The concept of Data mining is to draw out the concealed pattern as well as discover the relation among all features in a very large amount of data. Data mining techniques are used in a lot of areas, such as; education, marketing, engineering, finance, sport and medicine (Abdulla et al., 2015). Recently the assurance on data mining and educational systems is increased, this made the educational data mining a new area for research. Educational Data Mining $(\mathrm{EDM})$ is a very attractive area which contains many research domains that can handle the improvement of methods to use the data produced by educational context. Computational methodologies are used by

\begin{abstract}
Students' information in higher education institutions increases yearly. It is hard for them to extract meaningful information from the huge amount of data manually. Such information can support academic staff to stop students from dropping out at the end of courses. This can be done by evaluating the students' performance for the course and also by predicting their performance in the final exam early by using classification algorithms. Four classification algorithms, which are Decision Tree C4.5, Random research in order to classify and predict the students' performance. Furthermore, this research aimed at improving the Decision Tree C4.5 algorithm by adding a grid search function in order to improve prediction accuracy in classifying and predicting the students' performance. Also, the features of this evaluation have been extracted through the interviews with academic staff of three universities (University of Zakho, Duhok Polytechnic University and University of Duhok), in Duhok province, Kurdistan Region, Iraq and through the review of the literature. A new prototype has been proposed as a tool to classify and predict the students' performance by using Accrod.Net library. Three datasets were utilized in traditional $\mathrm{C} 4.5$ order to test the improved Decision Tree C 4.5 with the that the improved Decision Tree C4.5 outperformed the traditional C4.5 and also performed better when compared to C4.5 (J48) in Weka tool and other algorithms used in this research.
\end{abstract}

Keywords: Educational Data Mining, Classification Algorithms, Improved Decision Tree, Evaluation Methods, Accord.Net
EDM to evaluate the educational data. Additionally, educational data mining is a developed system which concentrates on developing the methods for extracting the relationship from a large amount of data that produced the domain of the education. These methods help in understanding how students learn along with understanding their behavior (Monjurul Alom and Courtney, 2018). In educational institutions, the students' performance is the most important issue in any educational process (Sivakumar et al., 2018). Hence, classifying and predicting student's' performance by using educational data mining techniques takes more attention by researchers according to the large amount of data that is generated yearly in educational institutions. Furthermore, the 
EDM is used to extract new hypotheses and discoveries about the performance of the students (Sivakumar et al., 2018).

EDM is continuously developing along with many data mining techniques used in educational environments. Therefore, the process of EDM techniques starts with discovering the relation among data by using classification, clustering, regression, association rule mining and others. Then, the trained model can be validated theoretically and after that, the trained model can be used to predict the output for new unseen data. Thus, the results of the prediction can be used to help teachers and students make the right decisions (Baker, 2010). The aforementioned techniques are responsible for exploring data from educational institutions which provide quality education regarding the students' performance as a main purpose (Baradwaj and Pal, 2012) Indeed, many researchers used EDM techniques in order to classify and predict the students' performance (Costa et al., 2017; Rao et al., 2017; Hussain et al., 2018; Kiu, 2018; Sivakumar et al., 2018).

Classification is one of the data mining techniques that is widely used in educational environments for classifying and predicting the students' performance in educational institutions (Baker, 2010). Classification is a supervised learning method used to put data into groups in order to aim at choosing classes. There are two models in the classification method; descriptive model and a predictive model. The descriptive model is responsible for distinguishing the records of different classes, while the predictive model is responsible for predicting classes for new unseen records (Pang-Ning et al., 2006). Many researchers have done their research in classifying and predicting the students' performance using classification algorithms. Based on the literature review, the most popular algorithms used by many researchers are Decision Tree C4.5, Random Forest, SVM and Naïve Bayes (Costa et al., 2017; Sivakumar et al., 2018).

In educational institutions, the data of the students increase yearly. This makes the teachers and researchers pay more attention to students' performance. Classifying and predicting the students' performance becomes a big duty and it is hard for them to do that manually (Rao et al., 2017). Furthermore, the issue of students' failure in courses becomes a very popular area nowadays for teachers and researchers (Costa et al., 2017). On the other hand, many researchers applied different classification algorithms in order to classify and predict the students' performance during their academic year. These algorithms were applied on datasets which contain features, such as: Academic features, socioeconomic and demographic features. Academic features include Marks, Quizzes, assignments, homework and so on (Mahboob et al., 2016; Kadambande et al., 2017;
Rao et al., 2017). Socio-economic and demographic features include gender, marital status, dormitory, family size and so on (Kostopoulos and Lipitakis, 2017; Rao et al., 2017). Moreover, some of these algorithms still need improvement as (Sivakumar et al., 2018) said "we improved the decision tree to get more effective accuracy results from the attribute values". This study tries to improve the Decision Tree C4.5 algorithm. This improvement can have more accuracy regarding the classification and prediction of the students' performance in the higher education institutes. In addition, researchers in this study used three other classification algorithms which are Random Forest, SVM and Naïve Bayes. These algorithms were used in order to see the comparison of the performance of these algorithms. However, there is no specific algorithm which performs better than others in solving problems (Tiago and Cheplygina,2014). Henceforth, we have chosen the most popular algorithms in such area based on our review. These algorithms are used in classifying the students' performance during the course and also used to early predict their performance in the final exam.

\section{Literature Review}

The term "Educational Data Mining" appeared for the first time in 2005, this happened during the 2005 annual conference of the Association for the Advancement of Artificial Intelligence in Pittsburgh, USA, in a workshop on Educational Data Mining. After the first workshop, EDM became popular and widely used in higher education, also many researchers have been working on this area in order to improve the quality of education (Romero and Ventura, 2007).

The data mining techniques presented by (Hussain et al., 2018), these techniques were used in higher education institutes for improving the students' academic performance to prevent them from dropping out. The aforementioned researchers collected data from three different colleges. These data consisted of demographic, socio-economic as well as academic information of three hundred students. Four classification algorithms; J48, Bayes Net, PART and random forest, were used in order to predict the student performance during their academic year. The results showed that Random Forest performed better than the others according to the accuracy of $99 \%$.

Online learning has a social impact on students' performance (Kiu, 2018). Also, the aforementioned study showed that there are many problems which make the experience of students destitute, such as: Less faceto-face interaction, low understanding, lack of concentration on learning activities, difficulty in performing teamwork activities and high social isolation. Furthermore, the level of understanding of students' 
learning can be understood and concentrated through knowledge discovered from students' event in their online learning. This knowledge can be used in order to predict the students' performance on their final degrees. In the previously mentioned study, a classification was done based on activities extracted from students' event logs in online learning, which also depends on their learning process, the above study theory was done by using a supervised leaning algorithm, J48. The results of the mentioned study illustrated that the model could improve the learning process of students and enhance their performance. Additionally, the generated model could give advice to students who were at risk in a timely manner.

Moreover, Sivakumar (2012) showed that activities in a learning environment and other activities, such as: Academic extracurricular, Co-Curricular activities, internal examinations and grade obtained in the university examination, affect the students' performance. In the aforementioned work, data were collected using the real-world dataset which was related to students' performance in education in India. This data contained information about the students including CGPA, History of arrears, lab performance and so on. In the same study, different data mining techniques, such as; Decision tree, Naive Bayes, Neural Network, KNN and Improved Decision tree, were used in order to predict the students' performance. The results of this experiment indicated that the improved decision tree performed better than the other classification algorithms.

Costa et al. (2017) claimed that the high rates of students' failure in courses of programming became a very interesting area for teachers and researchers to search for reasons. The strength of Educational Data Mining was presented in the aforementioned study in order to early predict the students' failure in programming courses (for problem statement with ref in overview). Furthermore, in the above study, four Algorithms: Naive Bayes (NB), Neural Network (NK), Decision Tree (DT) and Support Vector Machine (SVM) were used in order to predict student failure. These Techniques were applied on two data sources of Brazilian University, one came from the campus and the other came from distance education. The results presented that SVM performed better than the other techniques.

Students' data in educational institution increase yearly, this directs researchers' attention more towards the students' performance. Evaluating and predicting student's performance became a big task in the education system and it is hard to do that manually because of the databases which are multidimensional (Rao et al., 2017). For this purpose in this study, Educational Data Mining was utilized (EDM) to extract meaningful information from the huge amount of data. The aforementioned study depends on internal assessment, such as: Quiz, lab mark, class test and attendance. And also, two more features were added to the others; external assessments and demographics. Furthermore, in the above study, five data mining algorithms were used; Neural Network, Decision Tree, Support Vector Machine, Naive Bayes and KNearest Neighbor. The results showed that neural network performed better than others. The Neural Network accuracy was (98\%) followed by Decision Tree which was (91\%). Then, Support Vector Machine and KNearest Neighbor gave the same accuracy, which was $(83 \%)$. Lastly, the method that had lower prediction accuracy was Naïve Bayes by $(76 \%)$.

Kadambande et al. (2017) found that the students' education level is getting lower day by day which has become a large issue and therefore student performance prediction is important. For this purpose, in their study, two data mining techniques; semantic rules and Support Vector Machine (SVM), were used. The first technique (semantic rules) could deliver learning to students and also, could improve the quality content of education. This work helped the students who were at risk to avoid failure and help the good students improve their interest regarding education.

Abdallah et al. (2017) claimed that it has become easier to search data and get valuable knowledge from it because of the emergence of new technology which is enhanced by using data mining. In order to predict students' performance using their previous academic experience, data analytic techniques are applied to real case studies used in the previously mentioned research. A new hybrid classification technique was used which was based on fuzzy multi-criteria and decision tree classification. This technique is used in order to predict students' performance based on numerous principles such as school, age, family size, address, evaluation in previous grades and activities. In order to check the correctness of the model, the proposed method was compared with other famous classifiers. According to (Abdallah et al., 2017) study, this method is an assuring classification tool.

Kostopoulos and Lipitakis (2017) worked on predicting the student's performance (fail and pass) in the final exam of a distance learning undergraduate course in HOU. The effectiveness of active learning algorithms, J48 decision tree, JRip, Logistic Regression (LR), Multilayer Perceptron (MLPs), representative of Neural Networks, Naïve Bayes (NB) and Sequential Minimal Optimization (SMO), a very effective SVM algorithm, were examined in the aforementioned study. Early realization of the low performance of the students could lead to developing the personalized learning strategies accordingly with the students' need which also helps in enhancing the students' academic performance. The results illustrated that MLP algorithm outperformed the others with the accuracy of $81.09 \%$. 
Mahboob et al. (2016) Demonstrated that predicting students' performance is one of the most significant efforts in educational data mining. The study mentioned above indicated that it is possible to predict the success rate of the students, by using algorithms, such as Naïve Bayes, Random Forest and Decision Tree J48. The research mentioned above aimed to find the most useful features. These features effects on students' performance during their academic years which lead them to drop out. The result illustrated that the model was beneficial for predicting the students' performance. Furthermore, the Random Forest algorithm gave an accuracy with $100 \%$, J48 gave an accuracy with $93.3 \%$ and Naive Bayes gave an accuracy with $86.6 \%$.

Agaoglu (2016) showed that one of the most used tools in solving and understanding educational and administrative problems are data mining techniques in higher education. Research in educational data mining, focuses on building models which predict the students' performance. One of the most used tools in such models is the course evaluation. In this research, four classification tools; Support Vector Machine (SVM), Decision tree, discriminant analysis and Artificial Neural Network, were employed for evaluation and prediction. The results showed that the decision tree outperformed the others in terms of accuracy with $92.3 \%$. The benefits of this research illustrate the expressiveness and effectiveness of such models in higher education course evaluation, also, these benefits may be utilized to enhance the performance of the students and teachers.

Mueen (2016) employed three data mining classification techniques; Naïve Bayes, Decision Tree and Multilayer Perception, in order to predict and analyze the students' academic performance in a specific course. All these algorithms were applied on students' information, which were collected from two semesters of graduate students. The results showed that the Naïve Bayes algorithm performed better than the others in predicting with an accuracy of $86 \%$. The aforementioned study helps teachers find out the students who were expected to fail in the course and it also helps the instructors give more attention to students who are at risk and need to improve their performance.

Based on the previous works done, the most features used in their studies are illustrated in Table 1. In this research, the authors used EDM algorithms, such as: Decision Tree, Random Forest, SVM and Naïve Bayes, in order to evaluate the students' performance in a specific course, during the semester or academic year and also predict their performance in the final exam. In addition, this research proposed a system as a new tool for classification and prediction of students' performance in the higher educational institutes. Next, this research added a function to Decision tree $\mathrm{C} 4.5$ in order to get more accuracy regarding to the classification and prediction of the students' performance.
Table 1: The most common features used by previous studies

\begin{tabular}{ll}
\hline Features & \\
\hline Exams Marks & Children \\
Lab Work & Marital status \\
Assignment & Projects \\
Gender & seminars \\
Online Resources & Work \\
Quiz & Age \\
Dormitory & Participation \\
Attendance & Family size \\
\hline
\end{tabular}

\section{Classification Algorithms}

\section{C4.5 Decision Tree}

In the decision tree, Iterative Dichotomiser 3 (ID3) algorithm was created by Ross Quinlan in order to build a decision tree (Quinlan, 1986). Ross Quinlan Developed ID3 algorithm to $\mathrm{C} 4.5$ algorithm. Many researchers confirm that $\mathrm{C} 4.5$ is one of the most powerful algorithms in classification and outperforms others (Agaoglu, 2016; Hussain et al., 2018; Kiu, 2018). A recursive partitioning was used for building a decision tree. Divide and conquer is a general name for this process because it utilizes the values of the features to divide the data into smaller subsets of the same class. Furthermore, C4.5 can deal with discrete and continuous data, missing values, noise data and pruning tree after construction. C4.5 depends on entropy and gain ration in order to choose the best attribute as a root and then go on further splitting features.

\section{Random Forest}

Breiman (2001) has developed the Random Forest algorithm; both classification and regression can be done by Random forest. Random Forest can construct several decision trees in order to enhance the rate of classification and also to overcome the overfitting problem (Mahboob et al., 2016).

Random Forest is a data mining technique which utilizes the decision tree for classification. For creating a $\mathrm{K}$ number of not pruned trees, Random Forest each time chose a different part of the dataset. In Ran-dom Forest, the test data applied to all constructed trees and the most frequent output will be assigned to the tested data as a label (Mishra et al., 2014).

Random Forest has the idea of the real forest which says the more trees in the forest will have more robust. Also, Random forest will give the best accuracy if it has a higher number of trees in the forest. Random Forest does not pay attention to the number of trees in the forest, can handle missing values, never overfit and also can deal with categorical data. For measuring the purity and impurity of the features, Random Forest utilizes the Gini Index measurement. 


\section{Support Vector Machine (SVM)}

Support Vector Machine is a supervised machine learning algorithm that analyzes data for classification and regression. SVM assigns the input values to one of the classified class based on previous training data. In SVM, the classes are separated by a plane or hyperplane which maximizes the margins between data and minimizes the classification error (Agaoglu, 2016). There are three types of SVM; Hard margin SVM (Linear), Soft margin SVM (Linear with outliers) and Kernel SVM (Non-Linear) (Boser et al., 1992). Many studies used SVM in order to predict the students' performance (Costa et al., 2017; Kadambande et al., 2017; Rao et al., 2017), in which some of them the algorithm outperformed the others in terms of classification (Costa et al., 2017; Kadambande et al., 2017).

\section{Naive Bayes}

Naive Bayes techniques are a probabilistic classifier's simple family which is based on Bayes theorem with very powerful (Naive) independence assumption among all features. In machine learning, Naive Bayes uses the theory of probability that considers the effect of the value of the parameter of a given class (Pandey and Pal, 2011). Instead of a deterministic relationship where not always the same attribute values have the same output label, in Bayesian classifier the probabilistic relationship is considered among all classes and attributes. Attributes can be classified depending on its values, "can be expressed as the probability of a record of being from the class $\mathrm{Y}$, given that the record has a set of attributes $\mathrm{X}$. That is, $\mathrm{P}(\mathrm{Y}=\mathrm{y} \mid \mathrm{X}=\mathrm{x})$. A record is assigned to the class with the largest probability" (Reason, 2009). Additionally, Naive Bayes is highly scalable, fast, easily trained on a small dataset and can be used for both binary and multiclass classification. This technique was used in many studies, such as: (Costa et al., 2017; Rao et al., 2017; Sivakumar et al., 2018) and performed better than others in terms of classification in (Lopez Guarin et al., 2015).

\section{Research Methodology}

\section{Data Collection}

In this study, the data has been collected in two steps. In the first step, the most related works were reviewed by the researcher and find out what are the features used in their studies. Then, extracted the most common features used among them, which could be found in the survey area of this study. In the second step, the researchers interviewed (30) academic staff to get their ideas for selecting the most wanted and effected features on students' performance. These academic staffs were from computer science department in three universities (the University of Zakho, University of Duhok and Duhok Polytechnic University) in the Duhok province, Kurdistan Region, Iraq, as a case study. In the experimental study three datasets were used, two of them taken from academic staff as a case study and another one taken from the study of (Cortez and Silva, 2008) for more proof.

\section{Datasets}

\section{Dataset One}

The dataset contains a record of 73 students, each record with 9 features and one class label. The features are as follows: Assignment 5, practical 10, mid 25, mid 40, Absence, Gender, Part., Online Res and Dormitory. For more details (Table 2).

\section{Dataset Two}

The dataset contains a record of 74 students, each record with 9 features and one class. The features are as follows: G1, G2, Reports, Assignment, Absence, Gender, Part., Online Res and Dormitory. For more details (Table 3).

\section{Dataset Three}

The Cortez and Silva (2008) dataset contains many features with one class label.

Table 2: Students' dataset one

\begin{tabular}{lll}
\hline Attribute Name & Value & Description \\
\hline Attribute Name & Value & description \\
Assignment 5 & Numeric Value & The grades of assignments \\
Practical 10 & Numeric Value & Lab work marks of the students \\
Mid 25 & Numeric Value & Grades of midterm1 exam \\
Mid 40 & Numeric Value & Grades of midterm2 exam \\
Absence & High, Average, Low & How much the student be absents \\
Gender & Male, Female & Students' gender \\
Part. & Yes, No & participate in the class \\
OnlineRes. & Yes, No & $\begin{array}{l}\text { Does the student use online resources } \\
\text { or not }\end{array}$ \\
dormitory & Yes, No & If the student live in dormitory or not \\
Class & Pass, Fai & If the student will pass the final exam \\
& & or not in a specific subject. \\
\hline
\end{tabular}

Table 3: Students' dataset two

\begin{tabular}{lll}
\hline Attribute Name & Value & description \\
\hline G1 & Numeric Value & The grades of exam 1 \\
G2 & Numeric Value & The grades of exam 2 \\
Gender & Male, Female & Gender of students \\
Reports & Numeric Value & Grades of report of students \\
Assignment & Numeric Value & Grades of assignment given to students \\
Dormitory & Yes, No & If the student live in dormitory or not \\
Absence & High, Average, Low & How much the student be absents \\
OnlineRes. & Yes, No & If the student use the online resource or not \\
Practical & Numeric & Lab work marks of the students \\
Participation & Yes, No & If the student participate in the class \\
Class & Pass, Fail & The goal attribute to check if the student \\
& & will pass the final exam or not in a \\
& & specific subject. \\
\hline
\end{tabular}




\section{Improved Decision Tree C4.5}

In our improved algorithm we will use the same traditional C4.5, but we will add a grid search function which will allow the model to run more than one time in order to get the best model. The grid search is the function that tries to get the best combination of variables among a range of possible variables which produces the best fit model. For example, if there are two parameters, each has 10 values. Then the grid search will try to find the best model resulting in 100 models [29]. In other words, the grid search function uses a Cartesian product in order to find the combination variables; (Equation 1 to 3):

$$
\begin{aligned}
& A=\{1,2,3\} \\
& B=\{4,5,6\}
\end{aligned}
$$

$A \times B=\left\{\begin{array}{l}(1,4),(1,5),(1,6),(2,4),(2,5), \\ (2,6),(3,4),(3,5),(3,6)\end{array}\right\}$

Additionally, a grid search is a process of finding the best suitable parameter for the model based on the type of utilized model. Grid search can be applied to any machine learning algorithm in order to calculate the optimal parameters for an existing model. A grid search will construct a model for each combination point
(Evan Lutins, 2017). Thus, in our improved algorithm the grid search function will be used to find the best two parameters: BestJoin and MaxHeight parameters. The BestJoin parameter is responsible for deciding how many times each feature can participate in the decision tree process. The original algorithm explained that each feature can participate only once or could be repeated if it takes different values each time, but in many types of research, the researchers do not mention that and they do not even present their tree. Furthermore, in other research, such as: (Taruna and Pandey, 2014; Mahboob et al., 2016; Kiu, 2018), the researchers presented their tree but they never mentioned the feature participation and there are many features that had repeated more than one time. Thus, in our proposed method we decide to use it for learning the tree in order to find the best combination parameter. On the other hand, the MaxHeight parameter is responsible for allowing the growth of a tree at a specific height during learning. This parameter has not been mentioned before by any of the researchers in the literature of this study. For this reason, BestJoin and MaxHeight parameters are used in order to enhance the performance of the algorithm. Indeed, in the improved algorithm of $\mathrm{C} 4.5$ each set will be the value of BestJoin and MaxHeight respectively. Figure 1, the explanation of the process of this model is given.

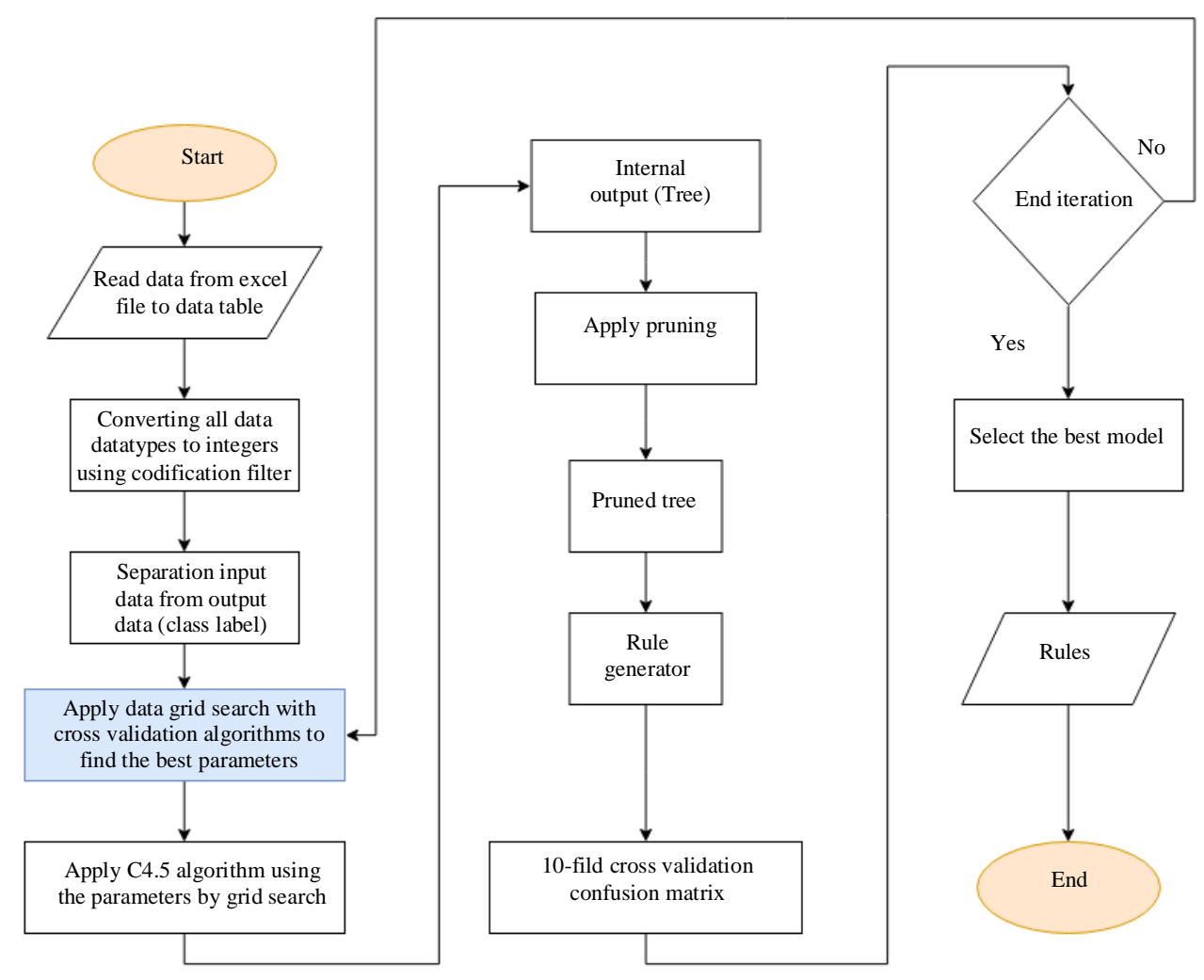

Fig. 1: C4.5 algorithm with data grid search 


\section{Proposed Prototype}

In this research, for classifying and predicating the students' performance, the researchers used a new tool which is called Acoord.Net library in visual studio by using c\#. Accord.Net is a scientific computation framework in .NET. The Accord.net framework contains many libraries that include a lot of scientific computing applications, such as: Machine learning, statistical data processing, pattern recognition, computer audition and computer vision. This framework presented a huge amount of hypothesis tests, support of popular performance measurement techniques, kernel functions and probability distribution. The Accord.Net library in visual studio was programmed by $\mathrm{C \#}$, which was constructed initially in 2010 and stabilized in October 2017 (Souza, 2017). In this proposed prototype (Fig. 2), the user can upload the dataset from excel sheets to the application. After that the user can select the class label and start applying the classification algorithms on that dataset.

\section{Evaluation Methods}

\section{Cross-Validation}

A cross-validation is a statistical tool used to evaluate the learning algorithms. In cross-validation, the data are split into two parts. The first part is the training part, which used to train the model. On the other hand, the second part is used to validate the model. The process of cross-validation depends on the cross over the training and validation data in the sequent round. This process used to give each data point a chance to be a validate data within the training data (Mankovskii et al., 2009).

One of the primary types of cross-validation is KFold cross-validation. Ten-Fold cross-validation is a form of K-Fold cross-validation and it is the standard method used to measure the error rate of a model. In tenfold cross-validation, the data is separated into ten equal folds and then nine folds will be used for training the model. On the other hand, the one remaining fold will be used for testing the model and the error rate will be calculated. This process will be repeated ten times, but each time will choose the different fold for testing the model without repetition. After that, the average error rate of all ten folds will be calculated (Kaufmann, 2005).

Table 4: Confusion matrix

\begin{tabular}{lllll}
\hline \multicolumn{2}{l}{ Predicted class } & & & \\
\hline Actual & Positive & TP & FN & P \\
Class & Negative & FP & TN & N \\
& Total & P' & N' & P + N \\
\hline
\end{tabular}

\begin{tabular}{|c|c|c|c|c|c|c|c|c|c|c|c|}
\hline \multirow[t]{2}{*}{ Algorithms } & \multicolumn{6}{|c|}{ Data Input and Class } & \multicolumn{5}{|c|}{ - $\square \times$} \\
\hline & & G1 & G2 & Reports & Assignment: & Gend 1 & \multirow{3}{*}{\multicolumn{2}{|c|}{$\begin{array}{l}\text { Variables } \\
\text { Number Of Samples }\end{array}$}} & & Correct & Incorrect \\
\hline Rules & b & 6 & 15 & 3 & 3 & M & & & 74 & 67 & 7 \\
\hline \multirow{2}{*}{ O No Pruning O Pruning } & & 16 & 12 & 3 & 3 & $\mathrm{~F}$ & & & & & \\
\hline & & 7 & 8 & 2 & 2 & M & \multicolumn{2}{|c|}{ Number Of Inputs } & 9 & & \\
\hline \multirow{2}{*}{$\gg \quad$ C4.5En Rules. } & & 14 & 1.5 & 2 & 2 & M & & & & & \\
\hline & & 12 & 13 & 3 & 3 & $\mathrm{~F}$ & \multirow{2}{*}{\multicolumn{2}{|c|}{ Number Of Outputs }} & 2 & & \\
\hline \multirow{2}{*}{$\gg$ Random Forest Rules } & & 2 & 14 & 3 & 3 & $F$ & & & & & \\
\hline & & 8 & 13 & 3 & 3 & M & \multirow{3}{*}{\multicolumn{2}{|c|}{ Classification Error }} & $0.0 \mathrm{~s}$ & 4285714 & 28571 \\
\hline \multirow{2}{*}{$\gg \quad$ Naive Bayes } & & 9 & 13 & 3 & 3 & M & & & & & \\
\hline & & 3 & 10 & 3 & 3 & M & & & & & \\
\hline \multirow{4}{*}{$\gg \quad$ SVM } & & 12 & 13 & 2 & 2 & $\mathrm{~F}$ & \multirow{2}{*}{\multicolumn{2}{|c|}{ Accuracy }} & \multirow{2}{*}{\multicolumn{3}{|c|}{0.905405405405405}} \\
\hline & & 18 & 15 & 3 & 3 & M & & & & & \\
\hline & & 15 & 12 & 4 & 4 & M & \multirow{3}{*}{\multicolumn{2}{|c|}{ Precision }} & \multirow{3}{*}{\multicolumn{3}{|c|}{$\begin{array}{l}0.891304347826087 \\
0.928571428571429\end{array}$}} \\
\hline & & 10 & 13 & 4 & 4 & M & & & & & \\
\hline \multirow{6}{*}{$\begin{array}{l}\text { C } 4.5 \text { Prune } \\
0.905405405405405\end{array}$} & & 3 & 7 & 3 & 3 & $F$ & & & & & \\
\hline & & 15 & 10 & 2 & 2 & $\mathrm{~F}$ & \multirow{4}{*}{\multicolumn{2}{|c|}{ Recall }} & \multirow{3}{*}{\multicolumn{3}{|c|}{\begin{tabular}{|l|}
0.953488372093023 \\
0.838709677419355
\end{tabular}}} \\
\hline & & 0 & 0 & 0 & 0 & M & & & & & \\
\hline & & 16 & 13 & 4 & 4 & $M$ & & & & & \\
\hline & & 15 & 15 & 4 & 4 & $F \quad v$ & & & \multirow{3}{*}{\multicolumn{3}{|c|}{\begin{tabular}{|l|}
0.921348314606742 \\
0.88135593220339
\end{tabular}}} \\
\hline & $<$ & & & & & & \multirow{2}{*}{\multicolumn{2}{|c|}{ F-Measure }} & & & \\
\hline \multirow[t]{5}{*}{ Highest Accuracy } & \multirow{5}{*}{\multicolumn{6}{|c|}{$\begin{array}{l}\text { Pass }=:(\mathrm{G} 1>4.5) \& 8(\mathrm{G} 2>8.5) \\
\text { Pass }=(\mathrm{G} 1>4.5) \& 8(\mathrm{G} 2<=8.5) \& 8 \text { (Reports }>2.5) \\
\text { Fail }=(\mathrm{G} 1<-4.5) \\
\text { Fail }=:(\mathrm{G} 1>4.5) \& \&(\mathrm{G} 2<=8.5) \& \& \text { (Reports }<=2.5)\end{array}$}} & & & & & \\
\hline & & & & & & & \multirow{4}{*}{$\begin{array}{l}\text { Select } \\
\text { File } \\
\text { Class }\end{array}$} & & & & \\
\hline & & & & & & & & Sheet2 & & & $\checkmark$ \\
\hline & & & & & & & & Class & & & $\checkmark$ \\
\hline & & & & & & & & & 100 & file & \\
\hline
\end{tabular}

Fig. 2: Proposed prototype 


\section{Confusion Matrix}

The confusion matrix is used to explain the performance of the classification model. In confusion matrix table the class values will be separated into positive $\mathrm{P}$ and negative $\mathrm{N}$. The variable True Positive (TP) used when the actual class is positive and also predicted as positive, while the variable False Negative (FN) is used when the actual class incorrectly predicted. On the other hand, the same idea is used for the negative class. The variable True Negative (TN) used when the actual class is negative and also predicted as negative, while the variable False Positive (FP) used when the class incorrectly predicted (Agaoglu, 2016). This information is given in Table 4.

Confusion matrix table can calculate Accuracy (Equation 1), Precision (Equation 2), Recall (Equation 3) and F-score (Equation 4). Furthermore, Accuracy is the measurement of total correct predictions among all predictions. Moreover, Precision is the measurement of total predictions marked as positive by the classifier, while Recall is the measurement of total positive classes that correctly predicted as positive F-score is a mean of Recall and precision (Hussain et al., 2018):

$$
\begin{aligned}
& \text { Accuracy }=(T P+T N) /(T P+T N+F N+F P) \\
& =(T P+T N) /(P+N) \\
& P=T P /(T P+F P) \\
& R=T P /(T P+F N) \\
& F=2(P R /(P+R))
\end{aligned}
$$

\section{Experimental Results}

In this research, the algorithms were applied on two datasets and also the algorithms were applied on another study dataset in order to make a comparison between improved Decision Tree C4.5 and the study method. In the next sections the results of all datasets will be shown.

\section{Results of Dataset One}

For this test, a dataset used that contains a records of 73 students, each record with 9 features. The features are as follows: Assignment 5, practical 10, mid 25, mid 40,
Absence, Gender, Part., Online Res and Dormitory. After applying the algorithms, the results shown as following; (Table 5 and Fig. 3).

After applying our machine learning algorithms to our dataset, the results become like those shown in Table 3 above. The best accuracy is given by improved $\mathrm{C} 4.5$ with $86 \%$. But the accuracy metric doesn't only mean that the model is good, it also means that the precision is needed in order to confirm that each instance in the dataset is classified with the same quality. Therefore, the better model has the highest accuracy with the highest precision. We applied a C4.5 (J48) algorithm from Weka tool and our normal $\mathrm{C} 4.5$ on our dataset, the results showed that the accuracy obtained by both tools was 82\%; Fig. 4. Our results compared to Weka tool results, our normal C4.5 algorithm performs better, like Weka tool's algorithm, but our improve C4.5 outperformed both in terms of accuracy and precision. For more details about the accuracy and other measurements (Table 6).

In the results, some of these algorithms could provide decision trees and rules, such as: C4.5 and improved C4.5. These trees and rules could be used to help the academic staff get a better understanding about what the main features are which have an effect on students' performance during the course. Furthermore, trees and rules help in predicting a class for new unseen records. In this way, academic staff and students can at least have an idea about what will happen to the students in the final exam before going to the exam depending on the students' performance during the course. Such rules will be show in Fig. 5 to 7 respectively.

Table 5: The results of dataset one

\begin{tabular}{lllll}
\hline Algorithm & Class & Precision & Recall & F-Measure \\
\hline C4.5 & Pass & 0.84 & 0.86 & 0.85 \\
& Fail & 0.79 & 0.76 & 0.77 \\
& Accuracy & & 0.82 & \\
Improved & Pass & 0.88 & 0.88 & 0.86 \\
C4.5 & Fail & 0.82 & 0.82 & 0.82 \\
& Accuracy & & 0.86 & \\
Random & Pass & 0.90 & 0.85 & 0.87 \\
Forest & Fail & 0.75 & 0.84 & 0.80 \\
& Accuracy & & 0.84 & \\
Naïve & Pass & 0.75 & 0.73 & 0.74 \\
Bayes & Fail & 0.58 & 0.60 & 0.59 \\
& Accuracy & & 0.71 & \\
SVM & Pass & 0.90 & 0.81 & 0.86 \\
& Fail & 0.68 & 0.83 & 0.75 \\
& Accuracy & & 0.82 & \\
\hline
\end{tabular}

Table 6: The improved C4.5 results Vs WEKA tool C4.5 results (data set one)

\begin{tabular}{lllll}
\hline Algorithm & Class & Precision & Recall & F-Measure \\
\hline Improved C4.5 & Pass & 0.88 & 0.88 & 0.86 \\
& Fail & 0.82 & 0.82 & 0.82 \\
WEKA Tool C4.5(J48) & Accuracy & 0.86 & 0.84 & 0.85 \\
& Pass & 0.86 & 0.79 & 0.78 \\
& Fail & 0.76 & 0.82 & \\
\hline
\end{tabular}



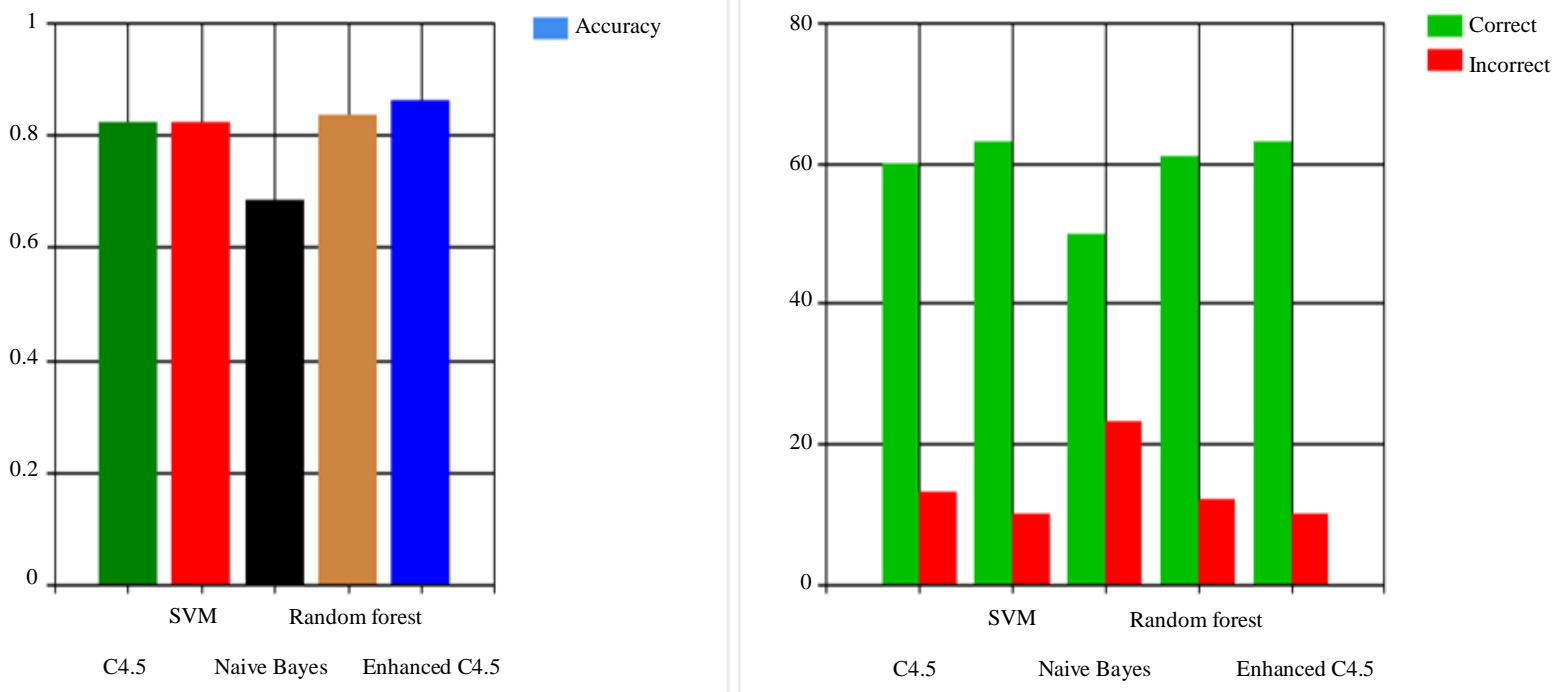

Fig. 3: Dataset one chart results

Weka Explorer

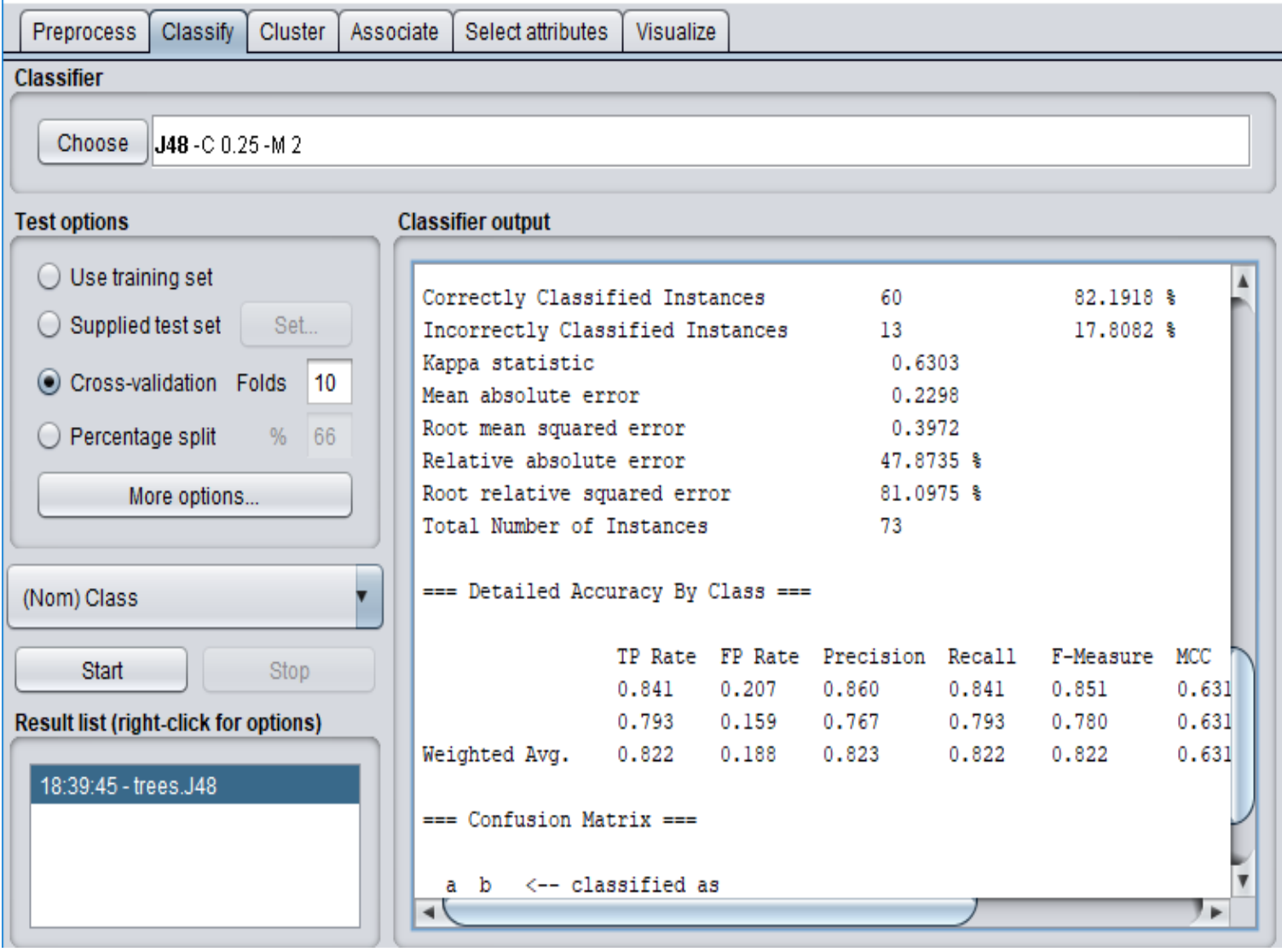

Fig. 4: Weka tool C4.5 results 
pass $=$ : (assignment $5<=1.5) \& \&($ practical $10>8.25)$

pass $=:($ assignment $5>1.5) \& \&(\operatorname{mid} 25>13.4375)$

pass $=$ : (assignment $5>1.5) \& \&(\operatorname{mid} 25<=13.4375) \& \&($ practical $10>8.75)$

pass $=$ : (assignment $5>1.5) \& \&(\operatorname{mid} 25<=13.4375) \& \&($ practical $10<=8.75)$

$\& \&(\operatorname{mid} 40<=12)$

pass $=$ : (assignment $5<=1.5) \& \&($ practical $10<=8.25) \& \&(\operatorname{mid} 25>9.0625)$

$\& \&($ Part. $==$ no $) \& \&($ Dirmitory $==$ no $)$

pass $=$ : (assignment $5<=1.5) \& \&($ practical $10<=8.25) \& \&(\operatorname{mid} 25>9.0625)$

$\& \&($ Part. $==$ no $) \& \&$ (Dirmitory $==$ yes $) \& \&(\operatorname{mid} 40>18.5)$

pass $=$ : (assignment $5>1.5) \& \&(\operatorname{mid} 25<=13.4375) \& \&($ practical $10<=8.75)$

$\& \&(\operatorname{mid} 40>12) \& \&$ (Dirmitory $==$ yes $) \& \&($ Part. $==$ yes $)$

pass $=$ : (assignment $5>1.5) \& \&(\operatorname{mid} 25<=13.4375) \& \&($ practical $10<=8.75)$

$\& \&(\operatorname{mid} 40>12) \& \&($ Dirmitory $==$ yes $) \& \&($ Part. $==$ no)

pass $=$ : (assignment $5>1.5) \& \&(\operatorname{mid} 25<=13.4375) \& \&($ practical $10<=8.75)$

$\& \&(\operatorname{mid} 40>12) \& \&($ Dirmitory $==$ no) $\& \&$ (Absence $<=1.5) \& \&$ (Part. $==$

yes $) \& \&($ Online Res. $==$ no $) \& \&($ Gender $==\mathrm{m})$

pass $=$ : $($ assignment $5>1.5) \& \&(\operatorname{mid} 25<=13.4375) \& \&($ practical $10<=8.75)$

$\& \&(\operatorname{mid} 40>12) \& \&$ (Dirmitory $==$ no) $\& \&$ (Absence $<=1.5) \& \&$ (Part. $==$

yes) $\& \&$ (Online Res. == no) \&\& (Gender == f)

pass $=$ : $($ assignment $5>1.5) \& \&(\operatorname{mid} 25<=13.4375) \& \&($ practical $10<=8.75)$

$\& \&(\operatorname{mid} 40>12) \& \&($ Dirmitory $==$ no) $\& \&($ Absence $<=1.5) \& \&($ Part. $==$

no) $\& \&($ Gender $==$ f) $\& \&$ (Online Res. == yes)

fail =: (assignment $5<=1.5) \& \&($ practical $10<=8.25) \& \&(\operatorname{mid} 25<=9.0625)$

fail $=$ : (assignment $5<=1.5) \& \&$ (practical $10<=8.25) \& \&(\operatorname{mid} 25>9.0625)$

$\& \&$ (Part. == yes)

fail $=$ : (assignment $5<=1.5) \& \&($ practical $10<=8.25) \& \&(\operatorname{mid} 25>9.0625)$

$\& \&$ (Part. == no) \&\& (Dirmitory == yes) \&\& $(\operatorname{mid} 40<=18.5)$

fail $=$ : $($ assignment $5>1.5) \& \&(\operatorname{mid} 25<=13.4375) \& \&($ practical $10<=8.75)$

$\& \&(\operatorname{mid} 40>12) \& \&($ Dirmitory $==$ no $) \& \&($ Absence $>1.5)$

fail $=$ : (assignment $5>1.5) \& \&(\operatorname{mid} 25<=13.4375) \& \&($ practical $10<=8.75)$

$\& \&(\operatorname{mid} 40>12) \& \&$ (Dirmitory $==$ no) $\& \&$ (Absence $<=1.5) \& \&$ (Part. ==

yes) \&\& (Online Res. == yes)

Fig. 5: C4.5 Rules without pruning

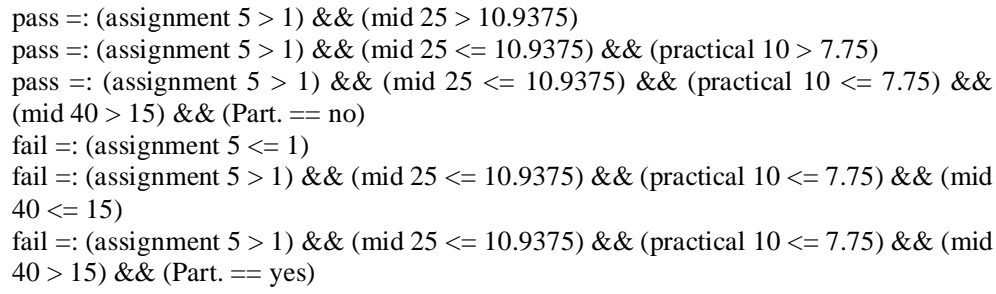

Fig. 6: C4.5 Results with pruning

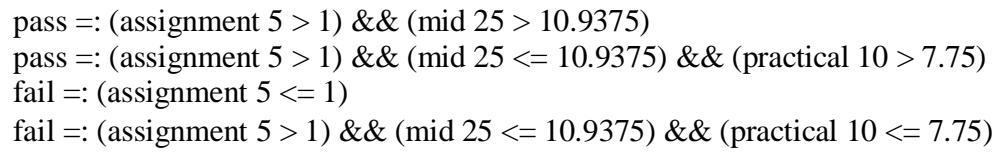

Fig. 7: Improved C4.5 rules

\section{Results of Dataset Two}

For this test dataset used that contains a records of 74 students, each record with 9 features and one class. The features are G1, G2, Reports, Assignment, Absence, Gender, Part., Online Res and Dormitory.
After applying the algorithms, the results shown as following (Table 7 and Fig. 8).

After applying machine learning algorithms used in this study, the results showed that Random Forest algorithm outperformed others, as shown in (Table 7 and Fig. 8) above. Since the main focus of this study is 
improving C4.5 algorithm, we applied our normal C4.5, improved C4.5 and C4.5 (J48) in WEKA tool on our dataset two. The results showed that the WEKA tool algorithm could give an accuracy of $89 \%$ (Fig. 9). On the other hand, our normal C4.5 algorithm could give an accuracy of $90 \%$ and improved $\mathrm{C} 4.5$ could give an accuracy of $95 \%$ (Table 8).

After the algorithms were applied on the second dataset, the following rules were shown from $\mathrm{C} 4.5$ and improved C4.5; Fig. 10 to 12 respectively.
Accuracy chart

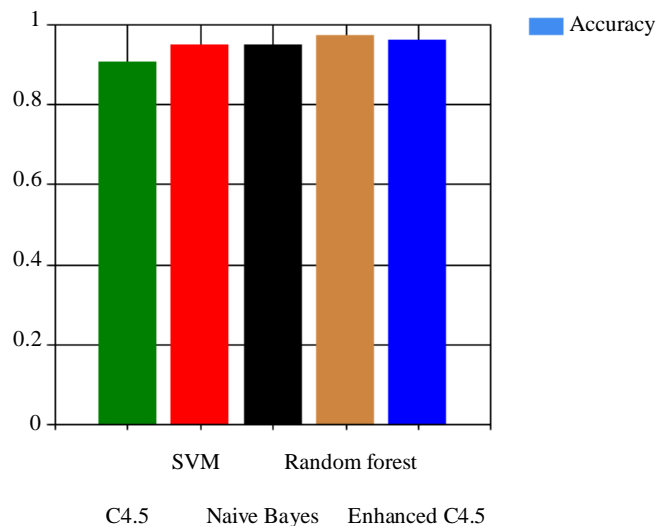

Variables

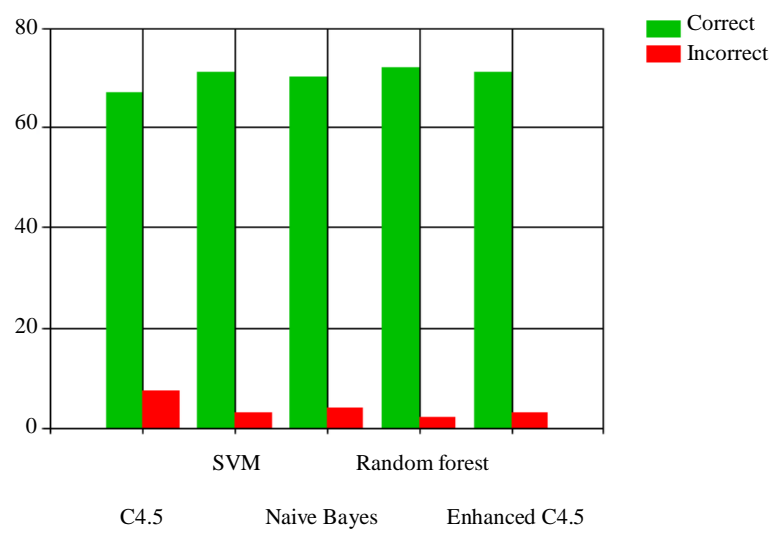

Fig. 8: Dataset two chart results

Q Weka Explorer

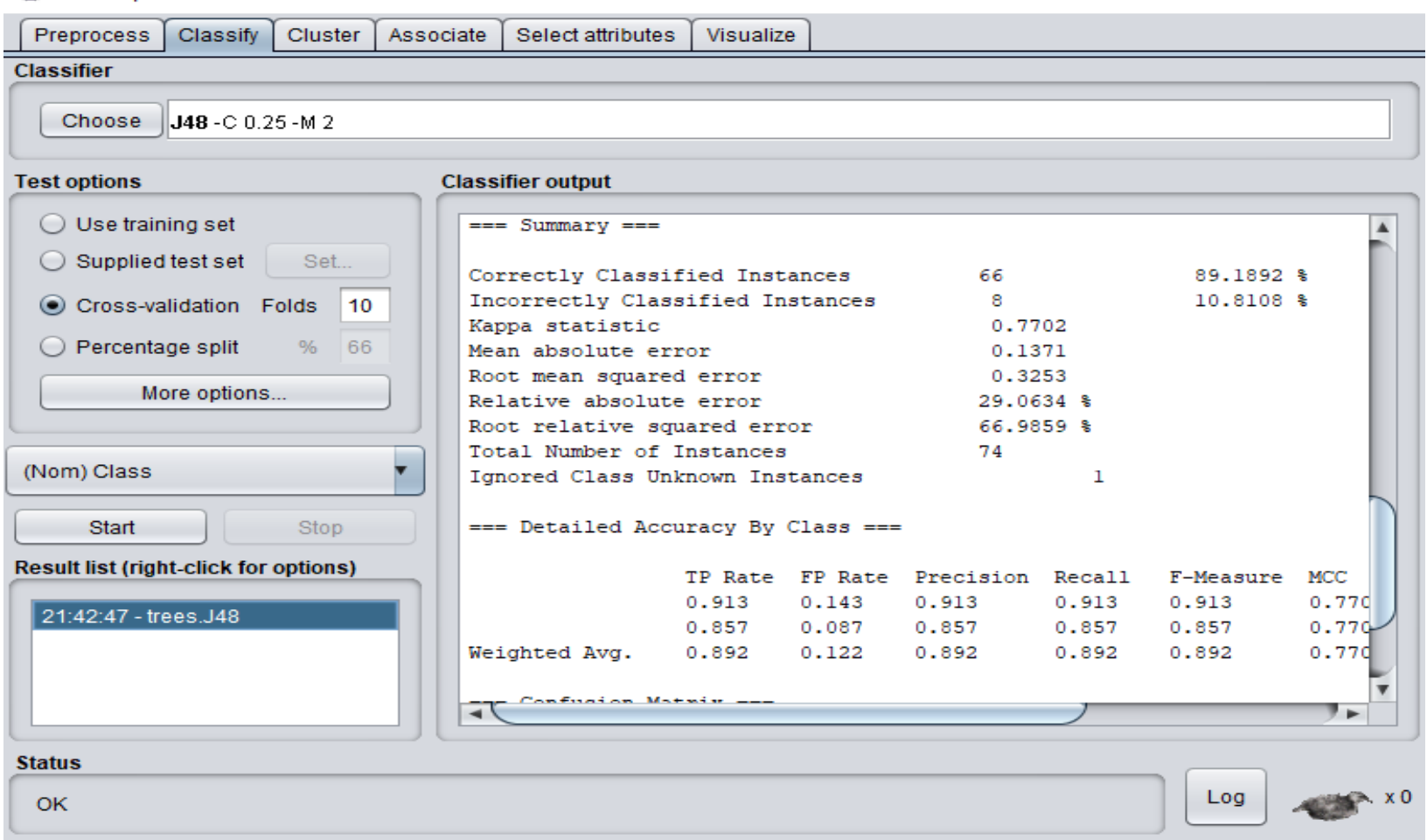

Fig. 9: C4.5 of WEKA tool result 
Pass $=:($ Reports $>2.5) \& \&(\mathrm{G} 1>4.5)$

Pass $=:($ Reports $<=2.5) \& \&(\mathrm{G} 1>10.5) \& \&(\mathrm{G} 2>3.5)$

Pass $=:($ Reports $>2.5) \& \&(\mathrm{G} 1<=4.5) \& \&(\mathrm{G} 2>10.5)$

Pass $=:($ Reports $<=2.5) \& \&(\mathrm{G} 1<=10.5) \& \&(\mathrm{G} 2>10.5) \& \&($ OnlinRes $==$ no $)$

Fail $=:($ Reports $<=2.5) \& \&(\mathrm{G} 1<=10.5) \& \&(\mathrm{G} 2<=10.5)$

Fail $=$ : $($ Reports $<=2.5) \& \&(\mathrm{G} 1>10.5) \& \&(\mathrm{G} 2<=3.5)$

Fail $=$ : $($ Reports $>2.5) \& \&(\mathrm{G} 1<=4.5) \& \&(\mathrm{G} 2<=10.5)$

Fail =: (Reports < $<2.5) \& \&(\mathrm{G} 1<=10.5) \& \&(\mathrm{G} 2>10.5) \& \&($ OnlinRes $==$ yes $)$

Fig. 10: C4.5 without pruning rules

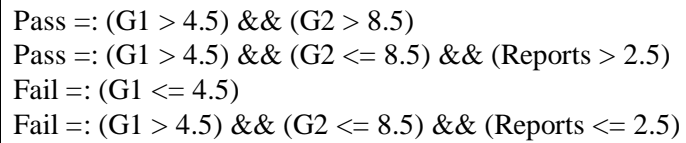

Fig. 11: C4.5 Pruned rules

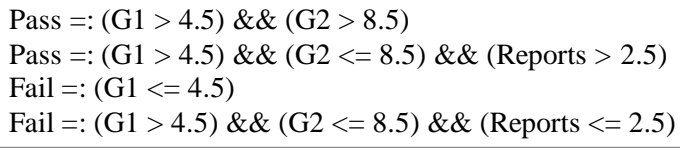

Fig. 12: Improved C4.5 rules

$$
\begin{gathered}
\text { FAIL }=:(\mathrm{G} 2<=9.5) \\
\text { PASS }=:(\mathrm{G} 2>9.5)
\end{gathered}
$$

Fig. 13: Improved C4.5 rules

\begin{tabular}{|c|c|c|c|c|}
\hline Algorithm & Class & Precision & Recall & F-Measure \\
\hline \multirow[t]{3}{*}{$\mathrm{C} 4.5$} & Pass & 0.89 & 0.95 & 0.92 \\
\hline & Fail & 0.92 & 0.83 & 0.88 \\
\hline & Accuracy & 0.90 & & \\
\hline \multirow[t]{3}{*}{ Improved C4.5 } & Pass & 0.95 & 0.97 & 0.96 \\
\hline & Fail & 0.96 & 0.93 & 0.94 \\
\hline & Accuracy & 0.95 & & \\
\hline \multirow[t]{3}{*}{ Random forest } & Pass & 0.97 & 0.97 & 0.97 \\
\hline & Fail & 0.96 & 0.96 & 0.96 \\
\hline & Accuracy & 0.97 & & \\
\hline \multirow[t]{3}{*}{ Naïve Bayes } & Pass & 0.97 & 0.93 & 0.95 \\
\hline & Fail & 0.89 & 0.96 & 0.92 \\
\hline & Accuracy & 0.94 & & \\
\hline \multirow[t]{3}{*}{ SVM } & Pass & 1.00 & 0.92 & 0.95 \\
\hline & Fail & 0.85 & 1.00 & 0.92 \\
\hline & Accuracy & 0.94 & & \\
\hline
\end{tabular}

Table 7: Dataset two results

Table 8: The improved C4.5 results Vs WEKA tool C4.5 results (data set two)

\begin{tabular}{lllll}
\hline Algorithm & Class & Precision & Recall & F-Measure \\
\hline Improved C4.5 & Pass & 0.95 & 0.97 & 0.96 \\
& Fail & 0.96 & 0.93 & 0.94 \\
C4.5 WEKA Tool & Accuracy & 0.95 & & 0.91 \\
& Pass & 0.91 & 0.85 & 0.91 \\
& Fail & 0.85 & & 0.85 \\
& Accuracy & 0.89 & &
\end{tabular}




\section{Results of Dataset Three}

This dataset were taken from UCI website of research (Cortez and Silva, 2008). After the proposed system algorithms applied on the aforementioned study dataset, the results showed that the improved Decision Tree C4.5 outperformed the aforementioned study Decision Tree, (Table 9 and 10).

The result of Cortez and Silva (2008) illustrated that Decision Tree can give an accuracy of $90 \%$ (Table 9).

Table 9: Results of Cortez and Silva (2008)

\begin{tabular}{llllll}
\hline Input Setup & NV & NN & SVM & DT & RF \\
\hline A & 0.91 & 0.88 & 0.86 & 0.90 & 0.91 \\
\hline
\end{tabular}

Table 10: The improved C4.5 results

\begin{tabular}{lllll}
\hline Algorithm & Class & Precision & Recall & F-Measure \\
\hline Improved C4.5 & Pass & 0.93 & 0.83 & 0.88 \\
& Fail & 0.90 & 0.96 & 0.93 \\
& Accuracy & 0.91 & & \\
\hline
\end{tabular}

On the other hand, the improved Decision Tree can give an accuracy of $91 \%$ (Table 10). Furthermore, the aforementioned study showed that G2 is the most important feature in the dataset. This is an improvement on our model because it could give the same result, (Fig. 13).

\section{Prediction Process}

Prediction is the process of selecting the right class label for new unseen records based on the trained data from the classification models. While the prediction process in Accord.Net Machine learning library is only dependable for predicting one by one record in form of a string. Thus, in this research, the prediction process begins by loading the excel file which contains the data that is wanted for prediction. Then the loaded data datatypes will be converted into integers, this converting is done in order for the system to make predictions for an unlimited number of records in sequence.

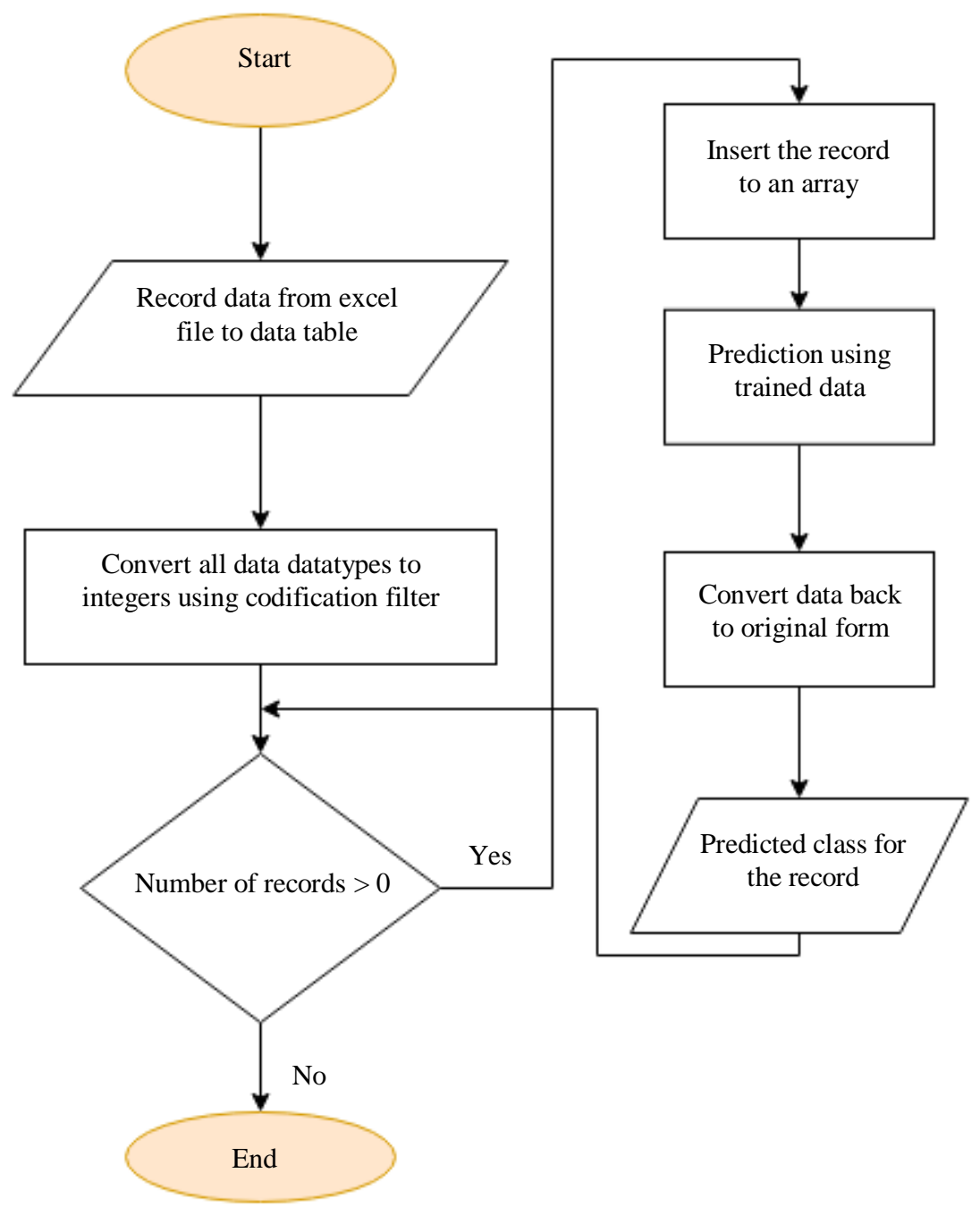

Fig. 14: The process of prediction 


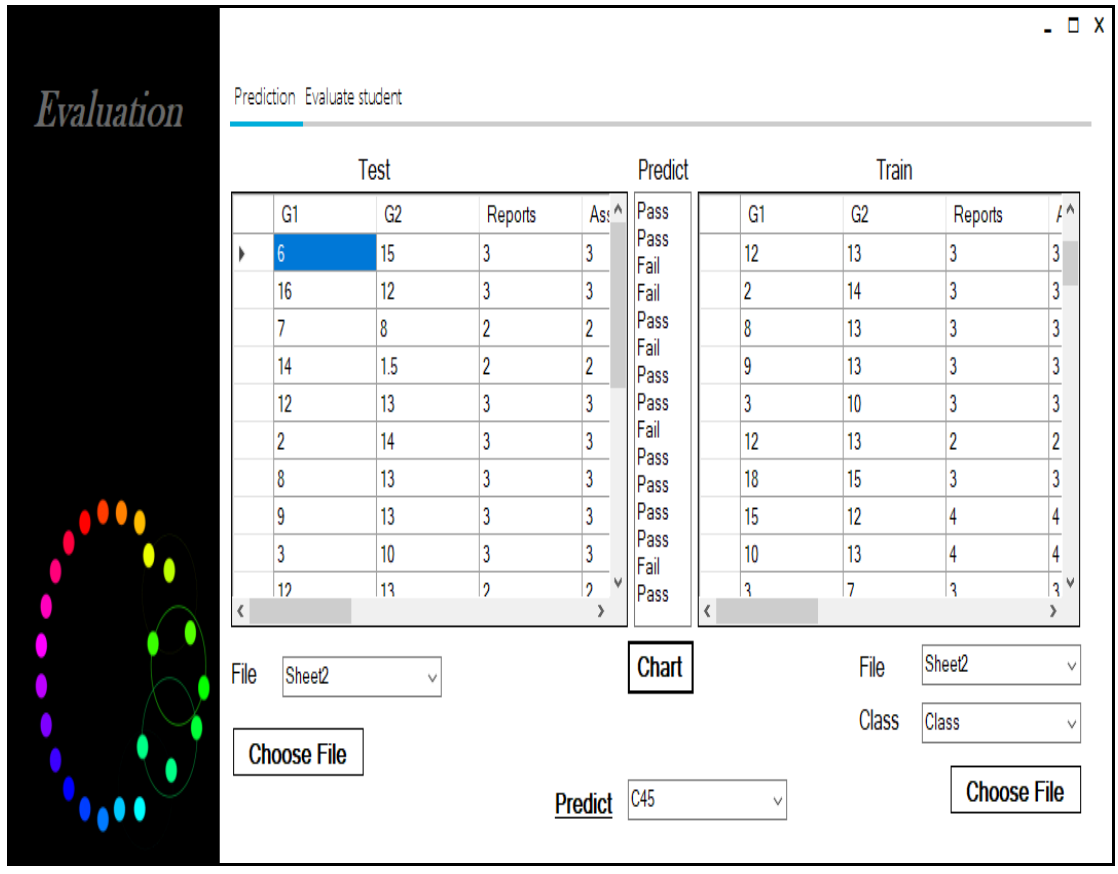

Fig. 15: The window of prediction

$-\square x$

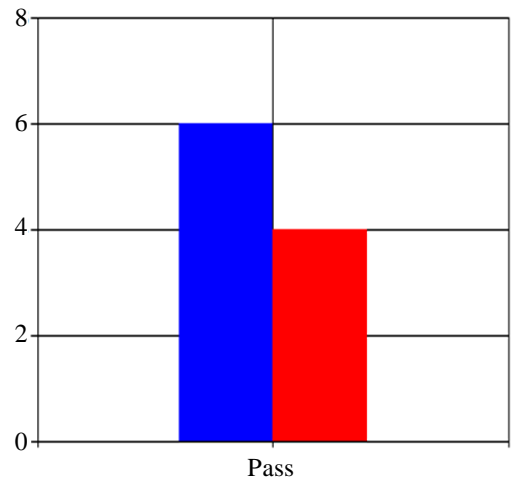

Fig. 16: The results of prediction

After that, the data will be loaded into an array one by one to test them on the trained data in order to get the class label. In Fig. 14, the flowchart of the prediction process will be provided to give a more clarification and in Fig. 15 and 16, the results of a ten-record test data will be shown

\section{Results’ Discussion}

After applying the classification algorithms on the two datasets (Dataset 1 and Dataset 2), the results showed that the improved Decision Tree C4.5 had performed better than standard $\mathrm{C} 4.5$ and also when compared to C4.5 (J48) in Weka tool.
Table 11: Comparison table

\begin{tabular}{llll}
\hline Algorithms & Dataset 1 & Dataset 2 & Dataset 3 \\
\hline Standard C4.5 & 0.82 & 0.90 & 0.90 \\
Weka C4.5 & 0.82 & 0.89 & 0.89 \\
Improved C4.5 & 0.86 & 0.95 & 0.91 \\
Random forest & 0.84 & 0.97 & 0.92 \\
Weka random forest & 0.83 & 0.94 & 0.91 \\
\hline
\end{tabular}

Furthermore, the improved Decision Tree C4.5 was applied to the dataset (Cortez and Silva, 2008) study. The results showed that the improved Decision Tree C4.5 had outperformed the Decision Tree C4.5 in the study mentioned in previous chapter. Table 11 gives more details about the comparison of performance of the algorithms used in this work.

\section{Conclusion}

The information of students in higher education institutions increases annually. It is difficult for them to take out meaningful information from huge amount of data manually. Such information can support academic staff in helping prevent students from dropping out at the end of courses. This can be achieved by evaluating students' performance for the course, as well as predicting their performance in the final exam early on by using classification algorithms. Four classification algorithms were used in this study; Decision Tree C4.5, Random Forest, Support Vector Machine (SVM) and Naive Bayes, in order to classify and predict the students' performance. Furthermore, this research aimed at 
improving the Decision Tree $\mathrm{C} 4.5$ algorithm by adding a grid search function in order to get more accuracy in classifying and predicting the students' performance. Also, the features of this evaluation were extracted through the interviews with academic staff and through the review of the literature. Three datasets were utilized in this research in order to test the improved Decision Tree C4.5 with traditional C4.5 and others. The results showed that the improved Decision Tree C4.5 outperformed the traditional $\mathrm{C} 4.5$ and also performed better when compared to $\mathrm{C} 4.5$ (J48) in Weka tool and other algorithms used in this research. Further research and improvements are required to generalize the proposed prototype to be a bedrock system of evaluating a big dataset of students in the educational field by researchers. Improving the system by adding unsupervised learning algorithms to support the unlabeled dataset or make the dataset ready for supervised learning algorithms.

\section{Acknowledgment}

This work was supported by the University of Duhok, University of Zakho and Duhok Polytechnic University. I would like to thank these universities for their help, support and contribution during the process of research investigation.

\section{Authors Contribution}

Mohammed Hikmat Sadiq: Participated in all works done in this research such as, Improving the decision Tree C4.5 algorithm by adding the grid search function and also helping in organizing question for interview in order to extract the most useful features related to students' performance in our institutions. Comparing results.

Nawzat Sadiq Ahmed: Participated in organizing the interview question for feature selection which related to students' performance, also he participated in organizing this article according to this journal conditions.

\section{References}

Abdallah, F.A., T. Anna and D. Babar, 2017. Analyzing students' performance using multi-criteria classification. Cluster Comput.

DOI: $10.1007 / \mathrm{s} 10586-017-0967-4$

Agaoglu, M., 2016. Predicting instructor performance using data mining techniques in higher education. IEEE Access, 4: 2379-2387. DOI: $10.1109 / A C C E S S .2016 .2568756$

Abdulla, N., Z. Ahmed and M. Gazzali, 2015. Measure Customer behaviour using C4.5 decision tree map reduce implementation in big data analytics and data visualization. Int. J. Innov. Res. Sci. Technol., 1: 228-235.
Baker, R.S.J., 2010. Data mining for education data mining for education advantages relative to traditional educational research paradigms.

Baradwaj, B. and S. Pal, 2012. Mining educational data to analyze student's performance. Int. J. od Advamced Comput Sci. Appl., 2: 63-69.

Breiman, L., 2001. Random forests. Mach Learn, 45: 532. DOI: 10.1023/A:1010933404324

Boser, B.E., I.M. Guyon and V.N. Vapnik, 1992. A training algorithm for optimal margin classifiers. Proceedings of the 5th Annual Workshop on Computational Learning Theory, Jul. 27-29, ACM, Pittsburgh, Pennsylvania, USA, pp: 144-152. DOI: 10.1145/130385.130401

Cortez, P. and A. Silva, 2008. Using data mining to predict secondary school student performance. Proceedings of the 5th Annual Future Business Technology Conference, (BTC' 08), Porto, pp: 5-12.

Costa, E.B., B. Fonseca, M.A. Santana, F.F. de Araújo and J. Rego, 2017. Evaluating the effectiveness of educational data mining techniques for early prediction of students' academic failure in introductory programming courses. Comput. Human Behav., 73: 247-256.

DOI: $10.1016 /$ j.chb.2017.01.047

Evan Lutins, 2017. Grid searching in machine learning: Quick explanation and python implementation. https://medium.com/@elutins/grid-searching-inmachine-learning-quick-explanation-and-pythonimplementation-550552200596

Hussain, S., N.A. Dahan, F.M. Ba-Alwib and N. Ribata, 2018. Educational data mining and analysis of students' academic performance using WEKA. Indones. J. Electr. Eng. Comput. Sci., 9: 447-459. DOI: 10.11591/ijeecs.v9.i2.pp447-459

Kadambande, A., S. Thakur, A. Mohol and P.A.M. Ingole, 2017. Predict student performance by utilizing data mining technique and support vector machine. Int. Res. J. Eng. Technol., 4: 2818-2821.

Kaufmann, M., 2005. Practical Machine Learning Tools and Techniques. 2nd Edn., Elsevier.

Kiu, C.C., 2018. Supervised educational data mining to discover students' learning process to improve students' Performance. In: Redesigning Learning for Greater Social Impact, Tang, S. and S. Cheah (Eds.), Springer, Singapore, pp: 249-258.

Kostopoulos, G. and A. Lipitakis, 2017. Engineering applications of neural networks.

Lopez Guarin, C.E., E.L. Guzman and F.A. Gonzalez, 2015. A model to predict low academic performance at a specific enrollment using data mining. Rev. Iberoam Tecnol. del Aprendiz, 10: 119-125. DOI: 10.1109/RITA.2015.2452632 
Mahboob, T., S. Irfan and A. Karamat, 2016. A machine learning approach for student assessment in elearning using Quinlan's C4.5, Naïve Bayes and random forest algorithms. Proceedings of the 19th International Multi-Topic Conference, Dec. 5-6, IEEE Xplore Press, Islamabad, Pakistan.

DOI: 10.1109/INMIC.2016.7840094

Mankovskii, S., M. Gogolla and S.D. Urban, 2009. Ontology Acquisition. In: Encyclopedia of Database Systems, Liu, L. and M.T. Özsu, (Eds.), Springer, Boston.

Mishra, T., D. Kumar and S. Gupta, 2014. Mining students' data for prediction performance. Proceedings of the 4th International Conference on Advanced Computing and Communication Technologies, Feb. 8-9, IEEE Xplore Press, Rohtak, India, pp: 255-262. DOI: 10.1109/ACCT.2014.105

Mueen, A., 2016. Modeling and predicting students' academic performance using data mining techniques. Int. J. Mod. Educ. Comput. Sci., 8: 36-42.

DOI: 10.5815/ijmecs.2016.11.05

Monjurul Alom, B.M. and M. Courtney, 2018. Educational Data Mining: A Case Study Perspectives from Primary to University Education in Australia. I.J. Information Technology and Computer Science, 2: 1-9.

DOI: $10.5815 /$ ijitcs.2018.02.01

Pandey, U.K. and S. Pal, 2011. Data mining: A prediction of performer or underperformer using classification. Int. J. Comput. Sci. Inform. Technol., 2: 686-690.

Pang-Ning, T., M. Steinbach and V. Kumar, 2006. Introduction to Data Mining, 1st Edn., Pearson Addison Wesley, ISBN-10: 0321321367, pp: 769.

Quinlan, J.R., 1986. Induction of decision trees. Mach. Learn., 1: 81-106. DOI: 10.1023/A:1022643204877
Rao, N.V.K., N. Mangathayaru and M.S. Rao, 2017. Evolution and prediction of radical multidimensional e-learning system with cluster based data mining techniques. Proceedings of the International Conference on Trends in Electronics and Informatics, May 11-12, IEEE Xplore Press, Tirunelveli, India, pp: 701-707.

DOI: 10.1109/ICOEI.2017.8300793

Reason, R.D., 2009. Student variables that predict retention: Recent research and new developments. NASPA J., 46: 482-501. DOI: 10.2202/19496605.5022

Romero, C. and S. Ventura, 2007. Educational data mining: A survey from 1995 to 2005 . Sci. Direct, 33: 135-146. DOI: 10.1016/j.eswa.2006.04.005

Sivakumar, R., 2012. A study on fuzzy and particle swarm optimization algorithms and their applications to clustering problems. Proceedings of the IEEE International Conference on Advanced Communication Control and Computing Technologies, Aug. 23-25, IEEE Xplore Press, Ramanathapuram, India, pp: 462-466. DOI: 10.1109/ICACCCT.2012.6320823

Sivakumar, S., S. Venkataraman and R. Selvaraj, 2018. Predictive Modeling of Students Performance using Improved Decision Tree. In: Advances in Electronics, Communication and Computing, Kalam, A., S. Das and K. Sharma (Eds.), Springer, Singapore, pp: 21-36.

Souza, C., 2017. Introduction-accord.NET machine learning in $\mathrm{C \#}$. http://accordframework.net/intro.html

Taruna, S. and M. Pandey, 2014. An empirical analysis of classification techniques for predicting academic performance. Proceedings of the IEEE International Advance Computing Conference, Feb. 21-22, IEEE Xplore Press, Gurgaon, India, pp: 523-528. DOI: 10.1109/IAdCC.2014.6779379

Tiago, A.A. and V. Cheplygina, 2014. How to decide the best classifier based on the data-set provided? 